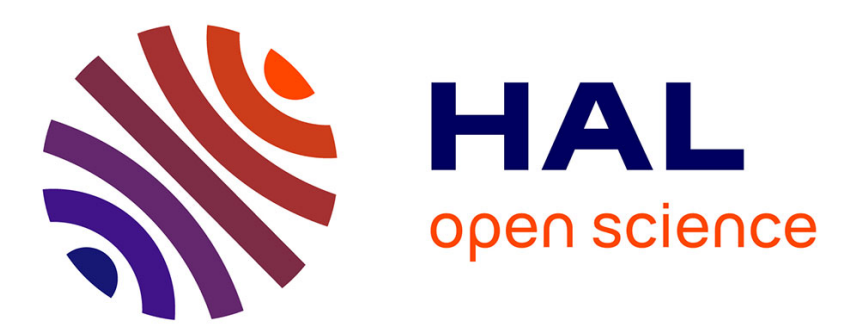

\title{
Dynamical model identification of population of oysters for water quality monitoring
}

Hafiz Ahmed, Rosane Ushirobira, Denis Efimov, Damien Tran, Jean-Charles Massabuau

\section{- To cite this version:}

Hafiz Ahmed, Rosane Ushirobira, Denis Efimov, Damien Tran, Jean-Charles Massabuau. Dynamical model identification of population of oysters for water quality monitoring. Proc. European Control Conference (ECC) 2014, Jun 2014, Strasbourg, France. hal-00986038

\section{HAL Id: hal-00986038 https://hal.inria.fr/hal-00986038}

Submitted on 30 Apr 2014

HAL is a multi-disciplinary open access archive for the deposit and dissemination of scientific research documents, whether they are published or not. The documents may come from teaching and research institutions in France or abroad, or from public or private research centers.
L'archive ouverte pluridisciplinaire HAL, est destinée au dépôt et à la diffusion de documents scientifiques de niveau recherche, publiés ou non, émanant des établissements d'enseignement et de recherche français ou étrangers, des laboratoires publics ou privés. 


\title{
Dynamical model identification of population of oysters for water quality monitoring
}

\author{
Hafiz Ahmed, Rosane Ushirobira, Denis Efimov, Damien Tran, Jean-Charles Massabuau,
}

\begin{abstract}
The measurements of valve activity in a population of bivalves under natural environmental conditions (16 oysters in the Bay of Arcachon, France) are used for a physiological model identification. A nonlinear auto-regressive exogenous (NARX) model is designed and tested. The model takes into account the influence of environmental conditions using measurements of the sunlight intensity, the moonlight and tide levels. A possible influence of the internal circadian/circatidal clocks is also analyzed. Through this application, it is demonstrated that the developed dynamical model can be used for estimation of the normal physiological rhythms of permanently immersed oysters and considered for detection of perturbations of these rhythms due to changes in the water quality, i.e. for ecological monitoring.
\end{abstract}

\section{INTRODUCTION}

Our world's environmental quality has been changing rather quickly and water quality everywhere is changing as well. Nowadays, national and international legislation has strict recommendations on protection of aquatic environment against the discharge of dangerous substances ${ }^{1}$. The realization of a protection program for aquatic systems needs a largely distributed network of ecological monitoring for water quality. Bio-indicators are increasingly used and show high efficiency, for instance in their ability to reveal the presence of low concentrations of contaminants through bioaccumulation in tissues ${ }^{2}$. However, if the goal is to sample at a high frequency, 24/7, with avoidance of missing short term events, most existing systems for ecological monitoring of aquatic environment are limited because they become rapidly too expensive and unrealistic. Indeed, frequently an intensive exploitation of human resources for collection of samples, chemical analysis and measurement of toxicity is involved [1]. A solution is to develop unmanned systems, able to work at high frequency by remote control, 24/7, either as bio-sensors by themselves (integrating information) or early warning detectors to trigger a sampling campaign. Today, networks of such online sensors, operating at large scale do not exist and are still a matter of research.

To fulfill that objective, an installation of numerous on-line remote sensors is required, working at high frequency for instant collection of information on a daily basis in marine environment [2]. Behavioral and physiological responses of wildlife to pollution are very sensitive and can be estimated for an indirect ecological monitoring. However, a limiting

\footnotetext{
${ }^{1}$ http://europa.eu/legislation_summaries/environment/water_protection_ management/128002b_en.htm;

http://water.epa.gov/scitech/swguidance/standards/criteria/aqlife/index.cfm;

${ }^{2}$ For example, see the US Mussel Watch, http://ccma.nos.noaa.gov/about/coast/nsandt/welcome.html.
}

factor today is that an on-line and continual physiological monitoring of wildlife requires transmission of high volumes of data, whose analysis needs sufficiently accurate models of animal behavior in natural conditions [3]. Other difficulties lie in the fact that animals may be heavily influenced by environment, group interactions and internal rhythms (e.g. feeding, breathing, spawning).

Observation of the opening and closing activities of bivalves is a possible way to evaluate their physiological behavior in reaction to environment. The deviations from a normal state can be used for detection of a contaminant in surrounding water. The pioneer work that analyzes bivalve's activities through the record of their valve movements (e.g., valvometry) was realized by Marceau [4] with smoked glazed paper. Today, valvometers are commercially available and are mainly based on the principle of electromagnetic induction, like the Mossel Monitor [5] or the Dreissena Monitor [6]. In recent years, the interest for modeling and estimation of behavior of marine animals directly in real marine conditions has intensively increased [7], [8], [9], [3].

A remarkable monitoring solution has been realized in the EPOC CNRS UMR laboratory in Arcachon, France [10], [11], [12], where a new framework for noninvasive valvometry has been developed and implemented since 2006 . The designed method is strongly based on bivalve's respiratory physiology and ethology. The obtained measurements have been deeply analyzed using statistical methods handling very large amount of data, which is the key to analyze the bivalve's behavior at a large scale. The obtained data is available on-line on the site of the project ${ }^{3}$. This system allows the bivalves to be studied in their natural environment with minimal experimental constraints. The obtained arrays of data of opening and closing activities of bivalves were used for analysis from different points of view using statistical approaches (estimation of probability density functions for time and amplitude of opening) [10], [11], [12].

The goal of the present study is the identification of a physiological dynamical model for bivalves using EPOC's data. A population of 16 oysters living in the Bay of Arcachon (France) is studied, the influence of external forces (like the sunlight and the moonlight, the tide level) are taken into account. Since the behavior of oysters is also guided by internal circadian/circatidal rhythms, their influence is also incorporated in the developed NARX model. The type and the origin of internal circadian/circatidal rhythms of bivalves is rather uncertain, that is why a side result of the

${ }^{3} \mathrm{http}: / /$ molluscan-eye.epoc.u-bordeaux 1.fr/ 
present study consists in verifying different hypotheses for modeling of circadian/circatidal rhythms for oysters. Van der Pol oscillator model has been used in this regard.

The outline of the paper is as follows. A brief description of the measurement scheme and experiments is given in Section 2. The model identification procedure is presented in Section 3. The verification of the model and its utility for an ecological monitoring of water quality are discussed in Section 4.

\section{Measurement System DESCRIPTION}

The monitoring site is located in the Bay of Arcachon, France, at the Eyrac pier (Latitude: $44^{\circ} 40 \mathrm{~N}$, Longitude: $\left.1^{\circ} 10 \mathrm{~W}\right)$. Sixteen Pacific oysters, Crassostrea gigas, measuring from $8 \mathrm{~cm}$ to $10 \mathrm{~cm}$ in length were permanently installed on this site. These oysters were all from the same age group (1.5 years old) and came from the same local supplier. They also all grew in the Bay of Arcachon. They were immersed on the sea bottom (at $3 \mathrm{~m}$ to $7 \mathrm{~m}$ deep in the water, depending on the tide activity).

The electronic equipment has been first described in [10],[13]. A considerable advantage of the developed monitoring system is that it is completely autonomous without in situ human intervention for one full year. Each animal is equipped with two light coils (sensors), $\approx 53 \mathrm{mg}$ each, fixed on the edge of each valve. These coils measure $2.5 \times 2.5 \times 2$ $\mathrm{mm}$ and were coated with a resin sealing before fixation on the valves. One of the coils emits a high-frequency sinusoidal signal, that is received by another coil. Measurements are performed every $0.1 \mathrm{sec}$ successively (with the frequency $10 \mathrm{~Hz}$ ) for each of the sixteen animals. This means that the behavior of a particular oyster is measured every 1.6 sec. Every day, 54000 triplets (1 distance, 1 stamped time value, 1 animal number) are collected for each oyster. The strength of the electric field produced between the two coils is proportional to the inverse of distance between the point of measurement and the center of the transmitting coil, which leads to estimation of the distance between coils. After some scaling, the obtained distance measurements characterize a relative opening of valves for an animal, i.e. a value between 0 and 100 is received, where 0 and 100 correspond to complete closing and opening in percentage respectively.

On the shore, a second electronic unit manages the data acquisition and transmission. This unit is equipped with a GSM/GPRS modem and uses Linux operating system for driving the first control unit immersed in the water, managing the data storage with a time stamp, accessing the Internet, and transferring the data. At the end of a working day (or any programmed experimental duration), the collected data is transmitted to a central workstation server located in the Marine Station at Arcachon, France. The valve activity data is stored in a central database. A schematic description of the monitoring system is presented in Figure 1.

The collected tidal data includes a measurement of the height of the water column every hour, while the times and levels of low and high tides are provided by the hydrographic

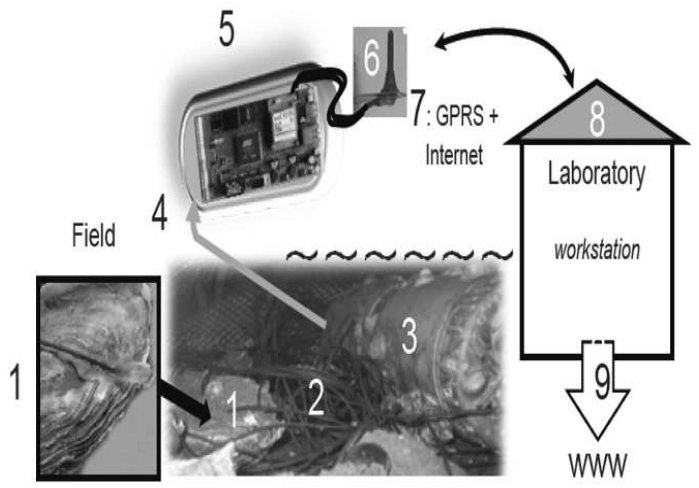

Fig. 1. [12] Synoptic representation of the system, from field to laboratory: (1) Oyster equipped with two electrodes; (2) connecting cables from oysters to first level electronic card; (3) first level electronic card in a waterproof case (immersed); (4) electrical connection between the first and the second electronic cards; (5) second electronic card (emerged); (6) GPRS antennae; (7) GPRS and Internet connection; (8) Marine Station of Arcachon; (9) daily update on Internet

and oceanographic service of the marine ${ }^{4}$. To take into account the external diurnal/nocturnal rhythms of the bivalve, the sun and the moon positions at the sampling site have been used. It is worth to note that data losses in transmission were sometimes observed due to impairment of data transfer.

\section{MODEL IDENTIFICATION}

Summarizing the description given in the previous section, after a specified duration of the experiment, the electronic system provides a matrix of values of relative opening of valves for each animal $P_{i, j} \in[0,100]$ for $1 \leq i \leq N$, $1 \leq j \leq n$, where $n=16$ is the number of oysters in the experiments and $N$ is the number of measured points, $N=54000 \times N_{\text {days }}$ with $N_{\text {days }}>0$ the number of days used for identification of the model. In our case, the data of 2007 collected at the Eyrac pier in Arcachon has been used, so $N_{\text {days }}=365$. In addition, the values $S_{i}, M_{i}, W_{i}$ are provided for $1 \leq i \leq N$ and characterize the sun and the moon positions with respect to the horizon in degrees and tide levels in meters respectively. The signals $S_{i}$ and $M_{i}$ take negative values for the corresponding positions below the horizon line.

Since $N \approx 2 \times 10^{7}$, to simplify computations and the presentation in this study, the measurements have been averaged on hourly basis, i.e. define $\delta N=\frac{3600[\mathrm{sec}]}{1.6[\mathrm{sec}]}=2250$

\footnotetext{
${ }^{4}$ http://www.shom.fr/
} 


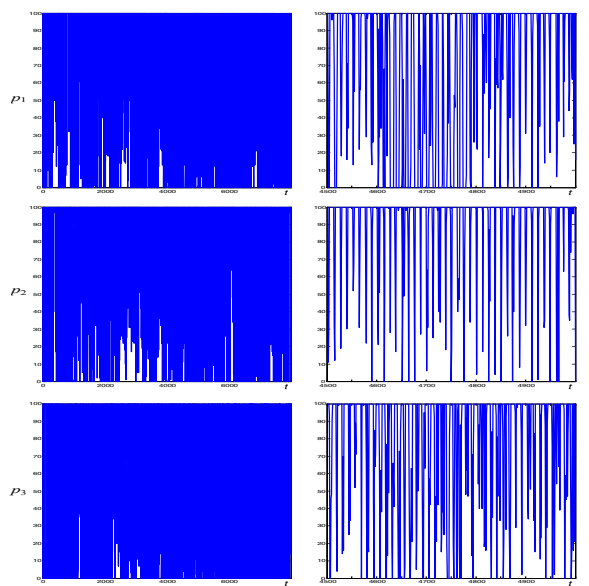

Fig. 2. Hourly averaged valve openings for three oysters

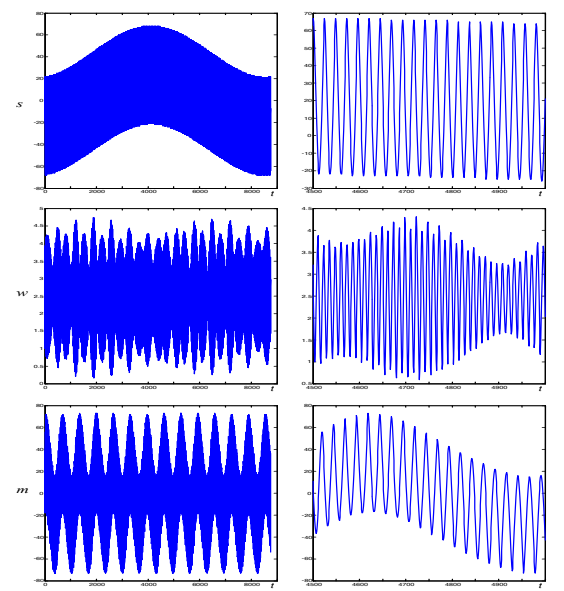

Fig. 3. Sun position, tide level and moon position

and $L=N / \delta N$, then for all $1 \leq k \leq L, 1 \leq j \leq n$ :

$$
\begin{aligned}
p_{k, j} & =\delta N^{-1} \sum_{q=(k-1) \delta N+1}^{k \delta N} P_{q, j}, \\
s_{k} & =\delta N^{-1} \sum_{q=(k-1) \delta N+1}^{k \delta N} S_{q}, \\
m_{k} & =\delta N^{-1} \sum_{q=(k-1) \delta N+1}^{k \delta N} M_{q}, \\
w_{k} & =\delta N^{-1} \sum_{q=(k-1) \delta N+1}^{k \delta N} W_{q},
\end{aligned}
$$

correspond to hourly average opening of valves, the sun and the moon positions, the water level respectively. Examples of obtained signals $p_{k, j}$ for three animals $(j=1,2,3), s_{k}, m_{k}$ and $w_{k}$ are shown in figures 2 and 3 with zoomed versions.

As we can conclude from these plots, different oysters have largely different behavioral activity and the correlation between them is rather weak. Define the averaged opening

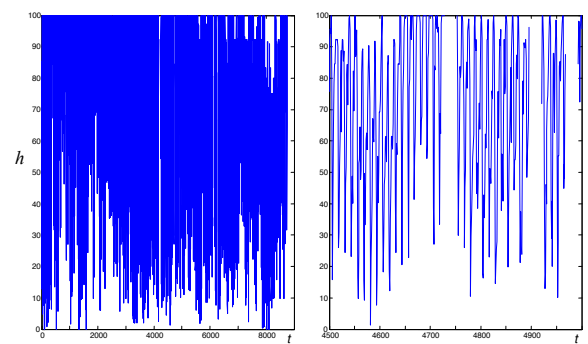

Fig. 4. Averaged hourly valve opening for the population

position of valves for the investigated population of oysters:

$$
h_{k}=n^{-1} \sum_{j=1}^{n} p_{k, j},
$$

the derived signal is presented in Figure 4. From this plot, we can deduce that the averaged on hourly basis openings of valves for population $h_{k}$ have a more regular and smoother behavior. This is a reason to consider the signal $h_{k}$ more suitable for the forthcoming analysis and model identification. Thus an averaged physiological population dynamics has to be modelled for oysters. A motivation of this choice is standard in modeling biological systems: each animal is largely perturbed by (stochastic) exogenous and endogenous forces, which are hard to predict or measure, while averaged signal $h_{k}$ is less sensitive to these perturbations and represents mainly the regular physiological behavior of the animals. Naturally, this is especially true if the population size $n$ is big, but this is not the case in the considered application due to various experimental complexities forcing to limit the population size to 16 .

It is well known [14] that the opening activity of oysters $h_{k}$ is externally governed by the sunlight, moon oscillations and the tide activity, denoted here by $s_{k}, m_{k}$ and $w_{k}$ respectively. Other sources of rhythmicity are internal (feeding, breathing etc.) and supervised by internal clocks. The reference signal generated by circadian rhythm genetics, which is directly influencing $h_{k}$, will be denoted by $c_{k}$. The generic structure of a dynamical physiological model for population of oysters can be presented as follows:

$$
\begin{aligned}
h_{k}= & F\left(h_{k-1}, \ldots, h_{k-\nu} ; s_{k}, \ldots s_{k-\mu} ;\right. \\
& \left.m_{k}, \ldots m_{k-\mu} ; w_{k}, \ldots w_{k-\mu} ; c_{k}, \ldots c_{k-\mu} ; \theta\right)+\epsilon_{k}, \\
c_{k}= & D\left(\xi_{k}\right), \\
\xi_{k}= & G\left(\xi_{k-1} ; s_{k}, \ldots s_{k-\mu} ; m_{k}, \ldots m_{k-\mu} ; w_{k}, \ldots w_{k-\mu}\right),
\end{aligned}
$$

where $\nu \geq 0$ represents the number of past events taken into account by the animals in order to determine the opening distance at the next time instant $k$ (i.e. in this model $k-1$ is the current time instant and $h_{k-1}$ is the current valve state); $\mu \geq 0$ is the number of previous values for the positions of sun/moon and tide levels that are used in the decision on the value of $h_{k}$ (the model (1) assumes that the instant values of $s_{k}, m_{k}$ and $w_{k}$ can be used for this decision); $\epsilon_{k} \in \mathbb{R}$ is the disturbance representing additional uncertain influencing forces and approximation errors (it is assumed 


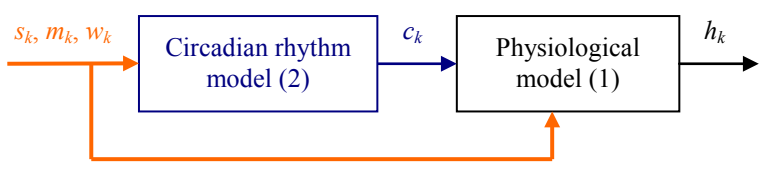

Fig. 5. Structure scheme of the generic model (1), (2)

that this term is sufficiently small if the model has been well identified); $\theta \in \mathbb{R}^{q}, q>0$ is the vector of constant parameters of the model (1), and $F: \mathbb{R}_{+}^{\nu+4 \mu+4+q} \rightarrow \mathbb{R}_{+}$is a function defining the physiological model structure; $\xi_{k} \in$ $\mathbb{R}^{p}$ is the state of the circadian oscillator (2); the functions $G: \mathbb{R}^{p+3 \mu+3} \rightarrow \mathbb{R}^{p}$ and $D: \mathbb{R}^{p} \rightarrow \mathbb{R}$ define the structure of the circadian rhythm model (2). The model (2) assumes that circadian oscillations are entrained by the external cues $s_{k}, m_{k}$ and $w_{k}$ (or some of them depending on the type of the rhythm under consideration, see below). The structure scheme of the model is given in Figure 5.

The identification procedure consists in selecting an appropriate structure for the physiological model $F(\cdot)$ and the circadian model $G(\cdot), D(\cdot)$, with posterior calculation of the corresponding vector of parameters $\theta$.

Further in this section two problems are considered. First, the issue of circadian rhythms modeling is discussed and a solution is proposed. Second, an ARMAX structure is selected for $F(\cdot)$ and the corresponding model is designed.

\section{A. Models of circadian clocks}

A circadian rhythm is a biological process that displays an endogenous and self-sustained oscillation with a period of about 24 hours. These rhythms are driven by a circadian clock.Circadian rhythms have been observed in almost all forms of living organisms. There exist many mathematical models of circadian clocks [15], [16], [17]. Different hypothesis on the nature of clocks presented in oysters [14] can also be issued, but not yet supported by a mathematical model. First, like many other animals, oysters may have oscillations with the period of 24 hours externally governed by sunlight (i.e. circadian clock). Second, they may also have a second exogenous rhythm with the period 12.4 hours and entrained by tides (circatidal clock). Third, the circadian clocks may be regulated by the moon with the period 24.8 hours (circalunidian clock). The diversity of hypothesis follows the variety of habitation areas of bivalves, e.g. arctic zones, ocean conditions with strong tides or oceans where tides are almost negligible. Finally, all these factors may entrain the animals simultaneously, and the circadian clock may have a varying period from 12.4 to 24.8 hours. In this work, for brevity, only the first hypothesis is considered, and the corresponding mathematical model is developed below.

Conventional models of circadian oscillators [15], [16], [17] have different complexity. There exist also methods to design generic oscillating systems [18] of any complexity. However, as it was observed in [19] and [20], any oscillating system on the limit cycle can be fully characterized by its current phase and by the form of the limit cycle (i.e. amplitude of oscillations). If the exact genetic or biochemical nature of circadian oscillations is not clear, then to preserve the model simplicity, the circadian rhythm can be represented by any oscillator, and a particular attention has to be paid to a relation of the phase of this oscillator and environmental rhythms. In [21] and [19], variants of Van der Pol oscillator [22] have been used for modelization of circadian rhythms for different animals. Van der Pol oscillator has a simple planar mathematical model with only two parameters that allows the form of the limit cycle and the period of oscillations to be tuned:

$$
\begin{aligned}
& \dot{\xi}_{1}=\alpha\left[\xi_{2}+\mu\left(\xi_{1}-\frac{4}{3} \xi_{1}^{3}\right)+u\right], \\
& \dot{\xi}_{2}=-\alpha \xi_{1},
\end{aligned}
$$

where $\xi_{1} \in \mathbb{R}$ and $\xi_{2} \in \mathbb{R}$ are the model states, $\alpha>0$ determines the model oscillation velocity (adjustment of this parameter regulates the period of oscillation for the model); $\mu>0$ is a parameter to determine the shape of oscillations ( $\mu \approx 0$ for almost harmonic oscillations, $\mu \geq 2$ leads to a nonlinear profile); $u \in \mathbb{R}$ is the model input that can be used for the entrainment. In our case the parameter $\alpha$ has to be selected in order to ensure the period of oscillations of 24 hours for the first hypothesis, for instance. The form of oscillations on the limit cycle, i.e. the value of parameter $\mu$, is less important since in the model (1), (2) the shape of $c_{k}$ can be taken into account later in the function $F(\cdot)$ and by tuning the parameters $\theta$ (the value $\mu=2$ is used in this work for all computations). Therefore, the functions $G(\cdot)$, $D(\cdot)$ in (2) can be defined based on Van der Pol equations discretized using the Euler method:

$$
\begin{aligned}
\xi_{1, k} & =\xi_{1, k-1}+T \alpha\left[\xi_{2, k-1}+\mu\left(\xi_{1, k-1}-\frac{4}{3} \xi_{1, k-1}^{3}\right)+u_{k}\right], \\
\xi_{2, k} & =\xi_{2, k-1}-T \alpha \xi_{1, k-1}, \\
c_{k} & =\max \left\{0, \xi_{1, k}\right\},
\end{aligned}
$$

where $T=1$ hour is the sampling time, $c_{k}$ is the positive part of $\xi_{1}$ and $u_{k}$ is the resetting input, which has to be specified.

The entrainment regulates the phase of circadian oscillations (synchronizes the exogenous cues and endogenous rhythms), the mechanism of entrainment and phase resetting can be well analyzed using the Phase Response Curve approach [20], [23]. For example, for the first hypothesis we select $u_{k}=\rho \max \left\{0, s_{k}\right\} / \max _{1 \leq k \leq L}\left|s_{k}\right|$, where $\rho>0$ is the scaling parameters that is selected in order to harmonize the amplitude of the input $u_{k}$ and the dimension of the limit cycle. The selection $\max \left\{0, s_{k}\right\}$ is applied since for the circadian rhythm the daylight is the principal zeitgeber, and the light is emitted when the sun is above the horizon only.

To generate the signal $c_{k}$ using the proposed model it is necessary to properly assign the initial phase for the circadian clock model (initial position on the limit cycle), which has to be coordinated with the current sun activity. A possible solution is to apply to the model the input $s_{s}$ pattern extracted from the first month of the experiment (or the last one in 
our case, since the experiment duration is 1 year and the sun position has annual periodicity) several times in order to entrain the oscillator.

\section{B. ARMAX model}

The ARMAX (auto-regressive-moving-average with exogenous inputs) model is one of the most popular structures used for identification in various fields of science [24]. Its advantages include linearity with respect to all signals and parameters, different effective methods for calculation of the parameters, simplicity of stability analysis of the obtained model and robustness with respect to perturbations. Therefore, the function $F(\cdot)$ in (1) is selected in the following form:

$$
\begin{aligned}
F(\cdot)= & \sum_{i=1}^{\nu} \theta_{i}^{h} h_{k-i}+\sum_{j=0}^{\mu} \theta_{i}^{s} \tilde{s}_{k-j} \\
+ & +\sum_{j=0}^{\mu} \theta_{i}^{m} \tilde{m}_{k-j}+\sum_{j=0}^{\mu} \theta_{i}^{w} w_{k-j}+\sum_{j=0}^{\mu} \theta_{i}^{c} c_{k-j} \\
\theta= & {\left[\theta_{1}^{h}, \ldots, \theta_{\nu}^{h}, \theta_{0}^{s}, \ldots, \theta_{\mu}^{s}, \theta_{0}^{m},\right.} \\
& \left.\ldots, \theta_{\mu}^{m}, \theta_{0}^{w}, \ldots, \theta_{\mu}^{w}, \theta_{0}^{c}, \ldots, \theta_{\mu}^{c}\right]^{T},
\end{aligned}
$$

where the positive values $\tilde{s}_{k}=\max \left\{0, s_{k}\right\}$ and $\tilde{m}_{k}=$ $\max \left\{0, m_{k}\right\}$ of the signals $s_{k}$ and $m_{k}$ respectively are used to model an influence of the sunlight and moonlight (the sun and the moon are above the horizon and deliver the light when $s_{k}$ and $m_{k}$ are positive only). Formally the proposed function $F(\cdot)$ is nonlinear with respect to its arguments $s_{k}$ and $m_{k}$ (it contains $\max \{\cdot\}$ ). The nonlinear model under consideration including the effect of circadian clock has the NARX structure (1), (2) for the selected $F(\cdot)$ and $G(\cdot), D(\cdot)$.

\section{VERIFICATION AND ANALYSIS}

Using the Least Square method [24], the estimates $\hat{\theta}$ of the parameters $\theta$ have been calculated.

$$
\hat{\theta}=\left(\Phi^{T} \Phi\right)^{-1} \Phi^{T} \Upsilon
$$

where $\Upsilon=\left[h_{\nu+1}, \ldots h_{L}\right]^{T}$ and the $j$-th row of the matrix $\Phi$,

$$
\begin{aligned}
\Phi_{j}= & {\left[h_{j+\nu-1}, \ldots, h_{j}, \tilde{s}_{j+\nu}, \ldots, \tilde{s}_{j+\nu-\mu}\right.} \\
& \tilde{m}_{j+\nu}, \ldots, \tilde{m}_{j+\nu-\mu}, w_{j+\nu}, \ldots, w_{j+\nu-\mu}, \\
& \left.c_{j+\nu}, \ldots, c_{j+\nu-\mu}\right]
\end{aligned}
$$

for all $1 \leq j \leq L-\nu$.

\section{A. Verification}

To evaluate the obtained accuracy of the designed model two performance costs $J_{\epsilon}$ and $J_{p}$ have been calculated. The cost $J_{\epsilon}$ estimates the average amplitude of $\epsilon_{k}$ (the approximation errors in the model):

$$
\begin{gathered}
\epsilon_{j}=h_{j+\nu}-\hat{h}_{j+\nu}, \hat{h}_{j+\nu}=\Phi_{j}^{T} \hat{\theta}, \quad 1 \leq j \leq L-\nu, \\
J_{\epsilon}=\sqrt{\frac{\sum_{j=1}^{L-\nu} \epsilon_{j}^{2}}{L-\nu}} .
\end{gathered}
$$
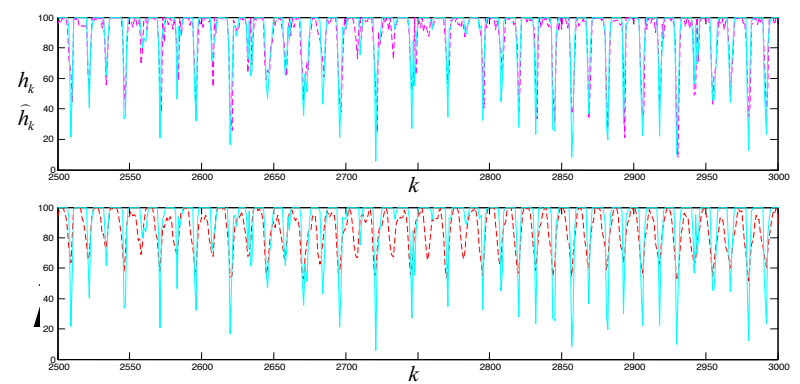

Fig. 6. The results of the model numerical verification

The cost $J_{p}$ evaluates the prediction quality of the model on an infinite time interval:

$$
\begin{gathered}
e_{j}=h_{j+\nu}-\tilde{h}_{j+\nu}, \quad 1 \leq j \leq L-\nu, \\
J_{p}=\sqrt{\frac{\sum_{j=1}^{L-\nu} e_{j}^{2}}{L-\nu}},
\end{gathered}
$$

where

$$
\begin{aligned}
\tilde{h}_{k}= & h_{k}, \quad 1 \leq k \leq \nu \\
\tilde{h}_{\nu+j}= & \sum_{i=1}^{\nu} \theta_{i}^{h} \tilde{h}_{\nu+j-i}+\sum_{z=0}^{\mu} \theta_{i}^{s} \tilde{s}_{\nu+j-z} \\
& +\sum_{z=0}^{\mu} \theta_{i}^{m} \tilde{m}_{\nu+j-z}+\sum_{z=0}^{\mu} \theta_{i}^{w} w_{\nu+j-z} \\
& +\sum_{z=0}^{\mu} \theta_{i}^{c} c_{\nu+j-z}, \quad 1 \leq j \leq L-\nu
\end{aligned}
$$

are the estimates of the valve positions $h_{k}$ generated independently by the designed model in the presence of the same inputs. Examples of the obtained estimates $\hat{h}_{k}$ and $\tilde{h}_{k}$ (for $\nu=\mu=72$, i.e. the oysters have 3 days of memory) are shown in Figure $6, J_{\epsilon}=12.06$ and $J_{p}=$ 23.18. As we can conclude from this example, the model demonstrates a sufficiently good accuracy of representation of the physiological behavior of oysters.

\section{B. Application to ecological monitoring}

Various problems related to an ocean ecological monitoring system are already mentioned in the introduction. Valve activity model of the oyster population can be used as a tool to overcome those problems. The area of population of bivalves is rather large, and the developed physiological model can serve for water quality monitoring, if we would compare the real measurements $h_{k}$ obtained by an embedded electronic unit on a bay and the estimates provided by the model $\tilde{h}_{k}$. For example, the following daily monitoring residual can be computed:

$$
r_{k}=\frac{1}{24} \sum_{z=0}^{23}\left|h_{k-z}-\tilde{h}_{k-z}\right|
$$

for all $24 \leq k \leq L$, where an averaging on 24 hours is used in order to decrease sensitivity of the residual with respect to measurement noises. For the dataset collected at 


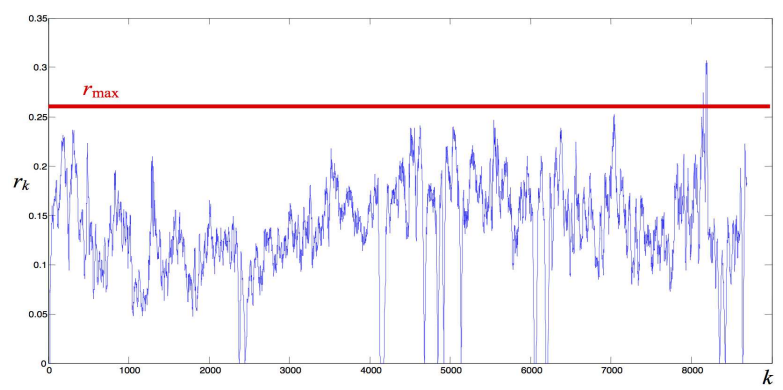

Fig. 7. Residual for water quality monitoring

the Eyrac pier in 2007, the obtained residual $r_{k}$ is shown in Figure 7. It is worth to stress that the Eyrac pier in the Bay of Arcachon is located in a fairly clean and well-protected area, but as we can see, the residual exceeds once the safety threshold $r_{\max }>0$, at the beginning of December 2007. It actually corresponds to a time period of heavy rain. Our calculation shows that oysters exhibited a strong deviation from their normal reference behaviour and this deviation has been registered by the proposed model based residual $r_{k}$. Since the output of a storm sewer is located at about 1015 meters from the oysters, we suggest that some unknown contaminant, washed by the rain, could have reach them. This simple case study justifies the utility of the developed approach for ecological monitoring.

\section{CONCLusion}

The paper presents the first development of a dynamical physiological model for oysters, which takes into account an influence of external rhythms (daylight, moonlight and tides) and internal circadian clocks as well. A generic "black-box" modeling approach has been used, and NARX structure of the model has been selected. The obtained model has been successfully applied for monitoring of water quality in the area of installation of the population of oysters, the obtained results are very encouraging.

In future works more sophisticated model structures have to be analyzed, and more hypothesis on the nature of circadian rhythms in bivalves have to be verified. The vector of inputs in the model can be extended by including the water temperature and the current cloudiness.

\section{REFERENCES}

[1] T. Telfer, H. Atkin, and R. Corner, "Review of environmental impact assessment and monitoring in aquaculture in europe and north america," in Environmental impact assessment and monitoring in aquaculture, ser. Fish Aquac Tech Pap No. 527, FAO. Rome: UN FAO, 2009, pp. 285-394.

[2] S. Kröger and R. Law, "Sensing the sea," Trends in Biotechnology, vol. 23, pp. 250-256, 2005.

[3] G. Galang, C. Bayliss, S. Marshall, and R. O. Sinnott, "Real-time detection of water pollution using biosensors and live animal behaviour models," in eResearch Australasia: emPower eResearch, Sydney, Australia, 2012.

[4] F. Marceau, "Contraction of molluscan muscle," Arch. Zool. Exp. Gen., vol. 2, pp. 295-469, 1909.

[5] K. J. M. Kramer, H. A. Jenner, and D. De Zwart, "The valve movement response of mussels: A tool in biological monitoring," Hydrobiologia, vol. 188/189, pp. 433-443, 1989.
[6] J. Borcherding, The Zebra Mussel Dreissena Polymorpha: Ecology, Biological Monitoring and First Applications in the Water Quality Management. NY: Gustav Fischer Verlag, 1992, ch. Another early warning system for the detection of toxic discharges in the aquatic environment based on valve movements of the freshwater mussel Dreissena polymorpha, pp. 127-146.

[7] H. Riisgard, J. Lassen, and C. Kittner, "Valvegape response times in mussels (mytilus edulis): Effects of laboratory preceding feeding conditions and in situ tidally induced variation in phytoplankton biomass," The Journal of Shellf ish Research, vol. 25, pp. 901-911, 2006.

[8] A. Robson, R. Wilson, and C. Garcia de Leaniz, "Mussels flexing their muscles: A new method for quantifying bivalve behavior," Marine Biology, vol. 151, pp. 1195-1204, 2007.

[9] J. Garcia-March, M. Sanchis Solsona, and A. GarciaCarrascosa, "Shell gaping behavior of pinna nobilis 1., 1758: Circadian and circalunar rhythms revealed by in situ monitoring," Marine Biology, vol. 153, pp. 689-698, 2008.

[10] D. Tran, P. Ciret, A. Ciutat, G. Durrieu, and J. Massabuau, "Estimation of potential and limits of bivalve closure response to detect contaminants: Application to cadmium," Environ. Toxicol. Chem., vol. 22, pp. 116-122, 2003.

[11] M. Sow, G. Durrieu, L. Briollais, P. Ciret, and J.-C. Massabuau, "Water quality assessment by means of hfni valvometry and high-frequency data modeling," Environmental Monitoring and Assessment, vol. 182, no. 1-4, pp. 155-170, 2011.

[12] F. G. Schmitt, M. De Rosa, G. Durrieu, M. Sow, P. Ciret, D. Tran, and J.-C. Massabuau, "Statistical study of bivalve high frequency microclosing behavior: Scaling properties and shot noise analysis," International Journal of Bifurcation and Chaos, vol. 21, no. 12, pp. 3565-3576, 2011.

[13] C. Chambon, A. Legeay, G. Durrieu, P. Gonzalez, P. Ciret, and J. Massabuau, "Influence of the parasite worm polydora sp. on the behaviour of the oyster crassostrea gigas: A study of the respiratory impact and associated oxidative stress," Mar. Biol., vol. 152, pp. 329338, 2007.

[14] D. Tran, A. Nadau, G. Durrieu, P. Ciret, J. P. Parisot, and J. C. Massabuau, "Field chronobiology in molluscan bivalves: How moon and sun cycles interactions deeply drive oyster activity rhythms," Chronobiol. Int., vol. 28, pp. 307-317, 2011.

[15] E. Klerman and M. St Hilaire, "On mathematical modeling of circadian rhythms, performance, and alertness," J Biol Rhythms, vol. 22, no. 2, pp. 91-102, 2007.

[16] P. Smolen, D. Baxter, and J. Byrne, "A reduced model clarifies the role of feedback loops and time delays in the drosophila circadian oscillator," Biophysical Journal, vol. 83, pp. 2349-2359, 2002.

[17] A. Goldbeter, "Computational approaches to cellular rhythms," Nature, vol. 420, pp. 238-245, 2002.

[18] D. Efimov and A. Fradkov, "Yakubovich's oscillatority of circadian oscillations models," Mathematical Biosciences, vol. 216, pp. 187191, 2008.

[19] D. Forger and R. Kronauer, "Reconciling mathematical models of biological clocks by averaging on approximate manifolds," SIAM Journal on Applied Mathematics, vol. 62, no. 4, pp. 1281-1296, 2002.

[20] D. Efimov, P. Sacre, and R. Sepulchre, "Controlling the phase of an oscillator: a phase response curve approach," in Proc. IEEE CDC 2009. IEEE, December 2009, pp. 7692-7697.

[21] M. Antle, D. Foley, N. Foley, and R. Silver, "Gates and oscillators: a network model of the brain clock," Journal of Biological Rhythms, vol. 18, no. 4, pp. 339-350, 2003.

[22] T. Kanamaru, "Van der pol oscillator," Scholarpedia, vol. 2, no. 1, p 2202, 2007.

[23] D. Efimov, "Phase resetting control based on direct phase response curve," Journal of Mathematical Biology, vol. 63, no. 5, pp. 855-879, 2011

[24] O. Nelles, Nonlinear System Identification. Berlin: Springer, 2000. 